\title{
Interstellar Dust and the Organic Inventories of Early Solar Systems
}

\author{
D. C. B. Whittet \\ New York Center for Studies of the Origins of Life, and Department of \\ Physics 6 Astronomy, Rensselaer Polytechnic Institute, Troy, \\ NY 12180, U.S.A.
}

\begin{abstract}
Interstellar dust grains are vectors for cosmic carbon and other biogenic chemical elements. They deliver carbon to protoplanetary disks in various refractory phases (amorphous, graphitic, etc.), and they are coated with icy mantles that contain organic molecules and water. The nature of the organics present in and on the dust appears to be closely related to physical conditions. Complex molecules may be synthesized when simple ices are irradiated. Astronomical observations show that this occurs in the vicinity of certain massive protostars, but it is not known whether our Solar System formed in such a region. Organic matter does not survive cycling though diffuse regions of interstellar space; any organics delivered to the early Earth must have originated in the parent molecular cloud, or in the solar nebula itself. A key question is thus identified: What was the star-formation environment of the Solar System? Possible constraints are briefly discussed.
\end{abstract}

\section{Introduction}

Asteroids, comets and interplanetary dust are thought to have delivered large quantities of volatiles and organic matter to the surface of the Earth during the early history of our Solar System, perhaps providing many of the raw materials needed to form our hydrosphere, biosphere, and life. The presence of enantiomeric excesses in meteoritic amino acids suggests the possibility that cosmic accretion might even have instigated homochirality of life on Earth (Bailey, this volume). Isotopic analyses demonstrate the presence of presolar grains in meteorites (Anders \& Zinner 1993), confirming that some of the interstellar dust entering the solar nebula survived and became incorporated into asteroidal parent-bodies. Comets also appear to be mixtures of nebular condensates and partially processed interstellar dust (Irvine et al. 2000).

Infrared spectroscopy provides the means of exploring the composition and evolution of dust in interstellar clouds and regions of active star formation. Grains composed of silicates and refractory carbon become coated with ices in dense interstellar clouds (see Whittet 2003 for an extensive review). The inventory of interstellar ices has been explored using data from the Infrared Space Observatory (Gibb et al. 2000). In cold, dark clouds, ices form principally by surface catalysis of atomic $\mathrm{H}, \mathrm{C}, \mathrm{N}$ and $\mathrm{O}$ adsorbed onto the dust, leading to species such as $\mathrm{H}_{2} \mathrm{O}, \mathrm{NH}_{3}$ and $\mathrm{CH}_{4}$; abundant gas-phase species such as $\mathrm{CO}$ may also freeze directly onto the dust. Carbon is present in a range of oxidation and 
hydrogenation states, from $\mathrm{CO}$ and $\mathrm{CO}_{2}$ to $\mathrm{CH}_{3} \mathrm{OH}$ and $\mathrm{CH}_{4}$. However, only in the environs of certain massive young stars is there evidence for the production of organics of higher molecular weight.

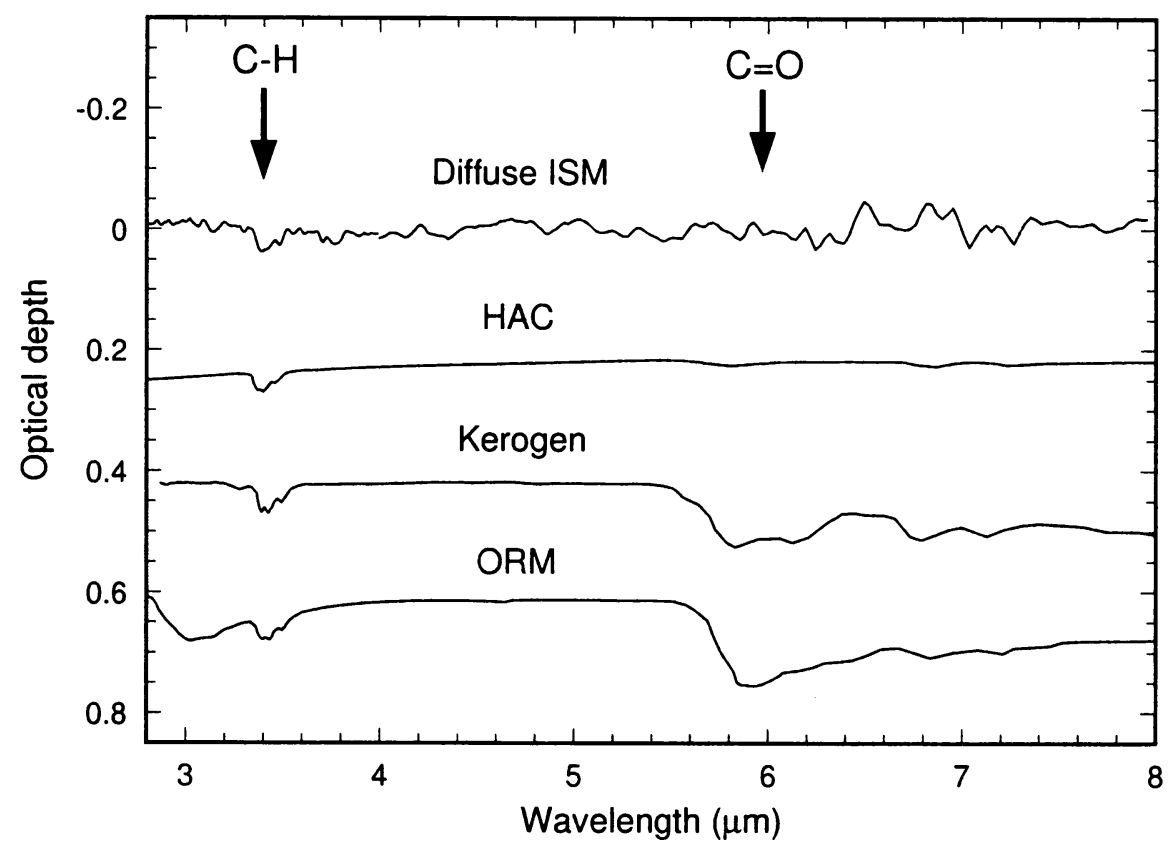

Figure 1. Infrared spectrum of the diffuse ISM (as observed toward the star Cyg OB2 no. 12; Whittet et al. 2001) compared with three laboratory analogs: hydrogenated amorphous carbon (HAC; Mennella et al. 1999), meteoritic kerogen (de Vries et al. 1993), and organic refractory matter (ORM) produced by UV photolysis of laboratory ices (Greenberg et al. 1995). The comparison spectra are scaled to match the observed depth of $\mathrm{C}-\mathrm{H}$ absorption at $3.4 \mu \mathrm{m}$.

\section{From Ices to Organics}

When luminous stars form within a dense cloud, they inject energy into their local environment that may induce both physical and chemical changes in the surrounding material. Mantled dust grains may be warmed, leading to partial or complete sublimation or crystallization of the ices, and their composition may be altered by radiatively-driven chemical reactions. Photon energies at the level of a few $\mathrm{eV}$ may break chemical bonds and convert saturated molecules such as $\mathrm{H}_{2} \mathrm{O}, \mathrm{NH}_{3}$ and $\mathrm{CH}_{4}$ into radicals. If irradiation is accompanied by warming, the radicals may be free to migrate through the mantles and react with other species. Laboratory simulations show that possible products include both kerogen-like organic polymers and prebiotic molecules such as nitriles and amino acids (e.g., Agarwal et al. 1985; Briggs et al. 1992; Bernstein et al. 1997, 2002). 


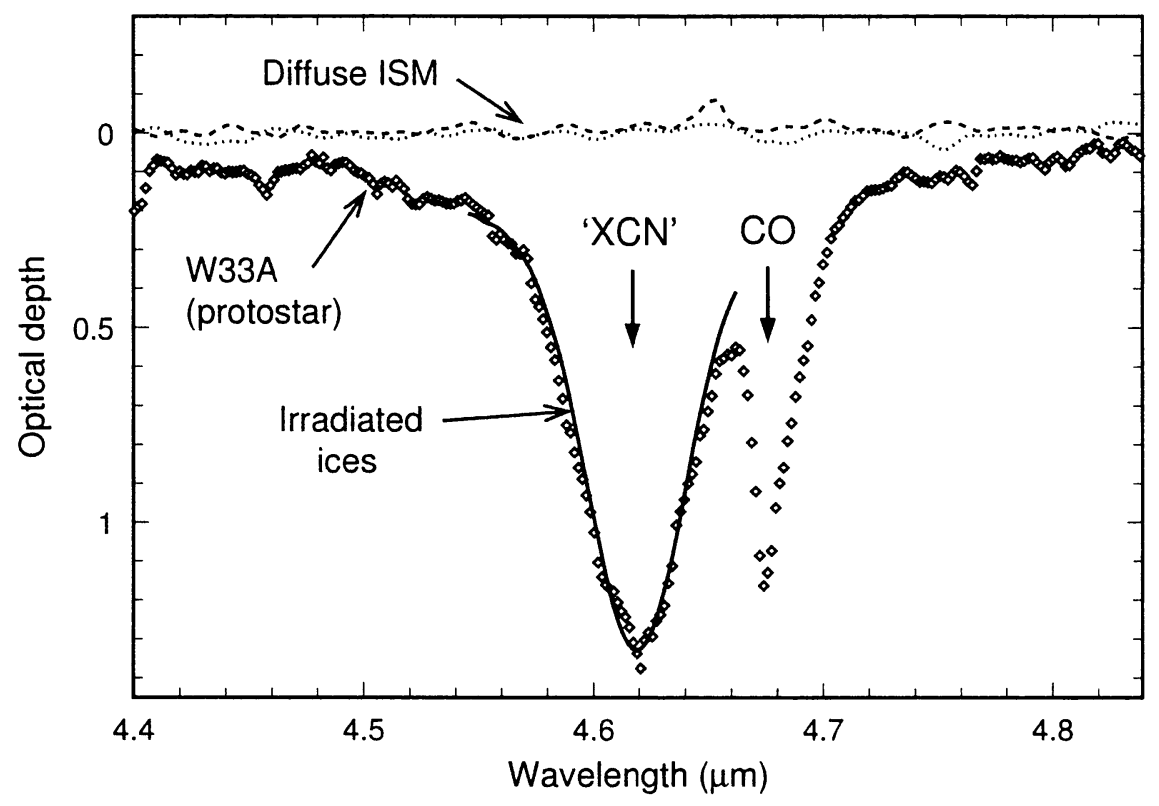

Figure 2. Spectrum of the luminous protostar W33A in the region of the vibrational mode associated with $\mathrm{C} \equiv \mathrm{N}$ groups in organic molecules (Gibb et al. 2000). Also shown for comparison are spectra of an object sampling the diffuse ISM (Cyg OB2 no. 12, dot/dash curves; Whittet et al. 2001) and of an ion-irradiated interstellar ice analog (solid curve overlaying the ' $\mathrm{XCN}$ ' profile; Palumbo et al. 2000).

One of the leading models for interstellar dust postulates that organic refractory mantles formed in this way survive for long periods in the general (diffuse) interstellar medium (Greenberg et al. 1995; Li \& Greenberg 1997). Figure 1 compares the spectrum of a star that samples "diffuse ISM" dust with three laboratory analogs: hydrogenated amorphous carbon (HAC), a kerogen extracted from the Murchison meteorite, and organic refractory matter (ORM) produced by photolysis of ices. An absorption feature at $3.4 \mu \mathrm{m}$ (the $\mathrm{C}-\mathrm{H}$ stretch) is present in each spectrum, but the ORM and kerogen also display a feature near $6 \mu \mathrm{m}$ (identified with carbonyl groups) that has no counterpart in the diffuse ISM. This and other evidence supports identification of the $3.4 \mu \mathrm{m}$ feature with HAC rather than ORM (Whittet 2003). The carbonyl feature has been detected in some protostellar spectra (where it is blended with the $\mathrm{H}_{2} \mathrm{O}$ bending mode; Gibb \& Whittet 2002), but it appears that the organics produced in the vicinity of such stars do not survive long-term outside of molecular clouds. Most likely, they are carbonized by continuous exposure to cosmic rays and energetic photons. It follows that any interstellar organics present in protoplanetary disks are relatively recent products of the parent cloud and not survivors from some earlier phase of star formation. 
The most exotic molecules are not generally produced in sufficient numbers to be detectable in the ices by infrared techniques (an alternative approach is to study their sublimation products at radio wavelengths in warm gas; see van Dishoeck \& Blake 1998). However, as a generic class, the $\mathrm{C} \equiv \mathrm{N}$-bearing molecules are a helpful exception, producing a spectral feature at $4.62 \mu \mathrm{m}$ observed in several protostars (Fig. 2); as the carrier lacks a specific identification, it is generally referred to as ' $\mathrm{XCN}$ ' (Pendleton et al. 1999). Molecules containing $-\mathrm{O}-\mathrm{C} \equiv \mathrm{N}$ (cyanate) groups appears to be favoured (Whittet et al. 2001). The position of the observed feature discriminates against nitriles (see Bernstein et al. $1997)$, and whilst isonitriles $(\mathrm{X}-\mathrm{N} \equiv \mathrm{C})$ give better wavelength coincidence, they are less stable than nitriles and should be less abundant.

\section{The Star Formation Environment of the Solar System}

Intense UV fields are predicted to occur only in regions containing high-mass stars (Adams \& Myers 2001). It is therefore not surprising that the cyanate and carbonyl features associated with organic products of ice irradiation are observed only in the spectra of protostars much more massive and luminous than the Sun. Objects thought to be reasonable analogs of the early Solar System generally yield no hint of them. Whilst processes within the solar nebula may have resulted in the production of some of the organics found in meteorites, the case for a significant presolar endowment of organics seems to depend on the star formation environment. Did the Sun form in a high-mass star formation region (an OB-association), where some of its neighbors may have been vigorous sources of UV radiation? The 'proplyd' region of Orion provides a graphic example of low-mass stars forming alongside high-mass neighbors. Or did it form in a quiescent dark cloud devoid of massive stars (a T-association), like that in Taurus? This is a difficult question to answer. Statistically, the odds seem to favor a T-association by about 10:1 (Adams \& Myers 2001)

One line of evidence that has often been discussed in this context is the abundance of short-lived radionuclides such as ${ }^{26} \mathrm{Al}$ in the early Solar System (e.g., Harper 1996; see Goswami \& Vanhala 2000 for a review). Excess radioactive parent isotopes are inferred from present-day abundances of their daughter products. Whilst some of these anomalies might arise from energetic processes within the Solar System (e.g., Shu et al. 2001), others appear difficult to explain without invoking an external source of freshly synthesized stellar material, such as ejecta from a supernova. As the relevant half-lives are $<2 \mathrm{Myr}$, the time available for this to occur is tightly constrained. The probability of the solar nebula being contaminated by supernova ejecta at the appropriate point in its development would have been vanishingly small if it formed in a T-association and had to rely on a random encounter with an unrelated progenitor. However, an $\mathrm{OB}$ association will naturally contain several progenitors that may become supernovae on timescales of $10 \mathrm{Myr}$ (Adams \& Myers 2001), short compared with the probable lifetime of the association. Star formation may therefore still be in progress when the most massive siblings reach the end of their lives.

Doubts remain as to the correct interpretation of the radionuclides anomalies. It will be very important in future research to attempt to dispel them, as this is our best window on the star-formation environment of the Solar System. 
Acknowledgments. The New York Center for Studies of the Origins of Life is a NASA-funded Specialized Center for Research and Training. The author gratefully acknowledges additional support from the NASA Exobiology program (grant NAG5-9148).

\section{References}

Adams, F. C., \& Myers, P. C. 2001, ApJ, 553, 744

Agarwal, V. K., et al. 1985, Origins of Life, 16, 21

Anders, E., \& Zinner, E. 1993, Meteoritics, 28, 490

Bernstein, M. P., Sandford, S. A., \& Allamandola, L. J. 1997, ApJ, 476, 932

Bernstein, M. P., Dworkin, J. P., Sandford, S. A., Cooper, G. W., \& Allamandola, L. J. 2002, Nature, 416, 401

Briggs, R., Ertem, G., Ferris, J. P., Greenberg, J. M., McCain, P. J., MendozaGomez, C. X., \& Schutte, W. 1992, Origins Life Evol. Biosphere, 22, 287

de Vries, M. S., Reihs, K., Wendt, H. R., Golden, W. G., Hunziker, H. E., Fleming, R., Peterson, E. \& Chang, S. 1993, Geochim. Cosmochim. Acta, 57,933

Gibb, E. L., \& Whittet, D. C. B. 2002, ApJ, 566, L113

Gibb, E. L., et al. 2000, ApJ, 536, 347

Goswami J. N. \& Vanhala H. A. T. 2000, in Protostars \& Planets IV, ed. V. Mannings et al. (Tucson: University of Arizona Press,),963

Greenberg, J. M., Li, A., Mendoza-Gomez, C. X., Schutte, W. A., Gerakines, P. A., \& de Groot, M. 1995, ApJ, 455, L177

Harper, C. L. 1996, ApJ, 466, 1026

Irvine, W. M., Schloerb, F. P., Crovisier, J., Fegley, B., \& Mumma, M. J. 2000, in Protostars \& Planets IV, ed. V. Mannings et al. (Tucson: University of Arizona Press), 1159

Li, A., \& Greenberg, J. M. 1997, A\&A, 323, 566

Mennella, V., Brucato, J. R., Colangeli, L., \& Palumbo, P. 1999, ApJ, 524, L71

Palumbo, M. E., Strazzulla, G., Pendleton, Y. J., \& Tielens, A. G. G. M. 2000, ApJ, 534, 801

Pendleton, Y. J., Tielens, A. G. G. M., Tokunaga, A. T., \& Bernstein, M. P. 1999, ApJ, 513, 294

Shu, F. H., Shang, H., Gounelle, M., Glassgold, A. E., \& Lee, T. 2001, ApJ, 548,1029

van Dishoeck, E. F., \& Blake, G. A. 1998, ARA\&A, 36, 317

Whittet, D. C. B. 2003, in Dust in the Galactic Environment $2^{\text {nd }}$ edition (Bristol: Institute of Physics Publishing)

Whittet. D. C. B., Pendleton, Y. J., Gibb, E. L., Boogert, A. C. A., Chiar, J. E., \& Nummelin, A. 2001, ApJ, 550, 793 


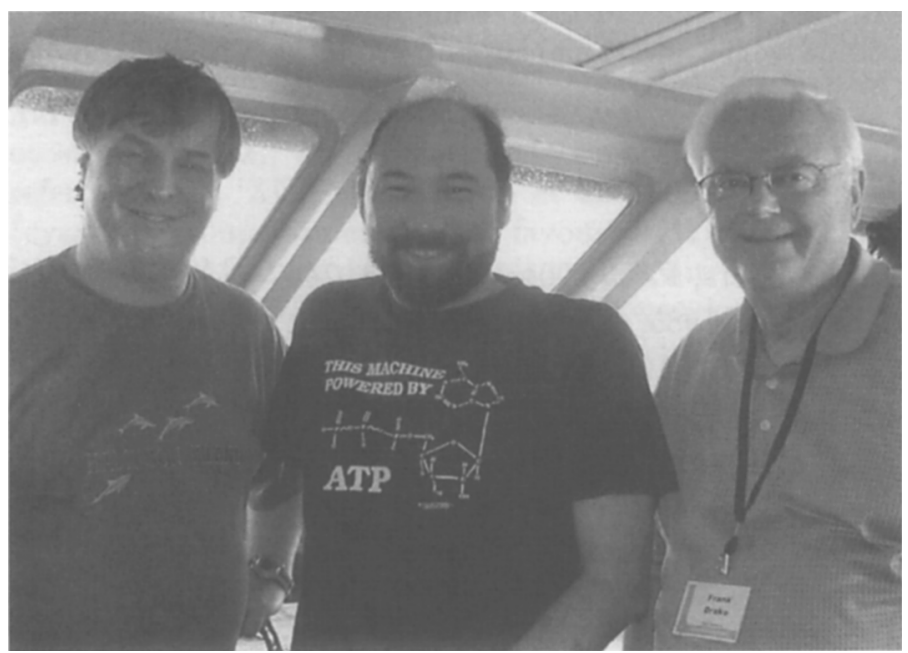

Ray Norris, Bill Welsh, \& Frank Drake (photo: Bill Welsh)

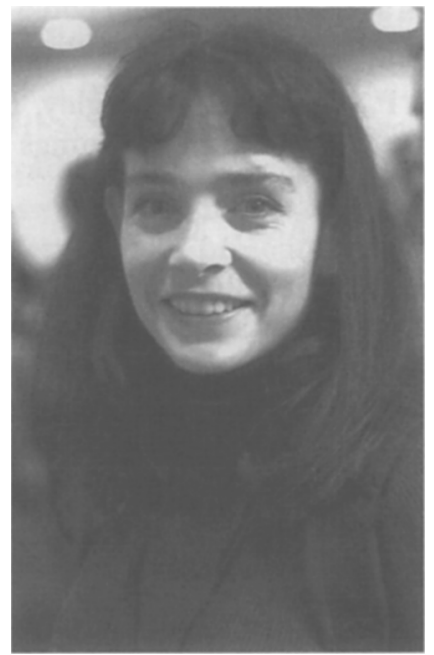

Abigail Allwood (photo: Seth Shostak) 